\title{
Fixed point and weak convergence theorems for point-dependent $\lambda$-hybrid mappings in Banach spaces
}

Young-Ye Huang ${ }^{1}$, Jyh-Chung Jeng ${ }^{2}$, Tian-Yuan Kuo ${ }^{3}$ and Chung-Chien Hong ${ }^{4^{*}}$

\author{
* Correspondence: chong@mail. \\ npust.edu.tw \\ ${ }^{4}$ Department of Industrial \\ Management, National Pingtung \\ University of Science and \\ Technology, 1 Shuefu Rd., Neopu, \\ Pingtung 91201, Taiwan \\ Full list of author information is \\ available at the end of the article
}

\begin{abstract}
The purpose of this article is to study the fixed point and weak convergence problem for the new defined class of point-dependent $\lambda$-hybrid mappings relative to a Bregman distance $D_{f}$ in a Banach space. We at first extend the Aoyama-lemoto-Kohsaka-Takahashi fixed point theorem for $\lambda$-hybrid mappings in Hilbert spaces in 2010 to this much wider class of nonlinear mappings in Banach spaces. Secondly, we derive an Opial-like inequality for the Bregman distance and apply it to establish a weak convergence theorem for this new class of nonlinear mappings. Some concrete examples in a Hilbert space showing that our extension is proper are also given.
\end{abstract}

2010 MSC: 47H09; 47H10.

Keywords: fixed point, Bregman distance, Gâteaux differentiable, subdifferential

\section{Introduction}

Let $C$ be a nonempty subset of a Hilbert space $H$. A mapping $T: C \rightarrow H$ is said to be

(1.1) nonexpansive if $\|T x-T y\| \leq\|x-y\|, \forall x, y \in C$, cf. [1,2];

(1.2) nonspreading if $\|T x-T y\|^{2} \leq\|x-y\|^{2}+2\langle x-T x, y-T y\rangle, \forall x, y \in C$, cf. [3-5];

(1.3) hybrid if $\|T x-T y\|^{2} \leq\|x-y\|^{2}+\langle x-T x, y-T y\rangle, \forall x, y \in C$, cf. [3,5-7].

As shown in [3], (1.2) is equivalent to

$$
2\|T x-T y\|^{2} \leq\|T x-y\|^{2}+\|x-T y\|^{2}
$$

for all $x, y \in C$.

In 1965, Browder [1] established the following

Browder fixed point Theorem. Let $C$ be a nonempty closed convex subset of a Hilbert space $H$, and let $T: C \rightarrow C$ be a nonexpansive mapping. Then, the following are equivalent:

(a) There exists $x \in C$ such that $\left\{T^{n} x\right\}_{n L \mathbb{N}}$ is bounded;

(b) $T$ has a fixed point.

The above result is still true for nonspreading mappings which was shown in Kohsaka and Takahashi [4]. (We call it the Kohsaka-Takahashi fixed point theorem.)

Recently, Aoyama et al. [8] introduced a new class of nonlinear mappings in a Hilbert space containing the classes of nonexpansive mappings, nonspreading mappings and hybrid mappings. For $\lambda \in \mathbb{R}$, they call a mapping $T: C \rightarrow H$

(C) 2011 Huang et al; licensee Springer. This is an Open Access article distributed under the terms of the Creative Commons Attribution License (http://creativecommons.org/licenses/by/2.0), which permits unrestricted use, distribution, and reproduction in any medium, provided the original work is properly cited. 
(1.4) $\lambda$-hybrid if $\|T x-T y\|^{2} \leq\|x-y\|^{2}+\lambda\langle x-T x, y-T y\rangle, \forall x, y \in C$.

And, among other things, they establish the following

Aoyama-Iemoto-Kohsaka-Takahashi fixed point Theorem. [8] Let $C$ be a nonempty closed convex subset of a Hilbert space $H$, and let $T: C \rightarrow C$ be a $\lambda$-hybrid mapping. Then, the following are equivalent:

(a) There exists $x \in C$ such that $\left\{T^{n} x\right\}_{n} \backslash \mathbb{N}$ is bounded;

(b) $T$ has a fixed point.

Obviously, $T$ is nonexpansive if and only if it is 0 -hybrid; $T$ is nonspreading if and only if it is 2-hybrid; $T$ is hybrid if and only if it is 1-hybrid.

Motivated by the above works, we extend the concept of $\lambda$-hybrid from Hilbert spaces to Banach spaces in the following way:

Definition 1.1. For a nonempty subset $C$ of a Banach space $X$, a Gâteaux differentiable convex function $f: X \rightarrow(-\infty, \infty]$ and a function $\lambda: C \rightarrow \mathbb{R}$, a mapping $T: C \rightarrow X$ is said to be point-dependent $\lambda$-hybrid relative to $D_{f}$ if

(1.5) $D_{f}(T x, T y) \leq D_{f}(x, y)+\lambda(y)\langle x-T x, f(y)-f(T y)\rangle, \forall x, y \in C$,

where $D_{f}$ is the Bregman distance associated with $f$ and $f(x)$ denotes the Gâteaux derivative of $f$ at $x$.

In this article, we study the fixed point and weak convergence problem for mappings satisfying (1.5). This article is organized in the following way: Section 2 provides preliminaries. We investigate the fixed point problem for point-dependent $\lambda$-hybrid mappings in Section 3, and we give some concrete examples showing that even in the setting of a Hilbert space, our fixed point theorem generalizes the Aoyama-Iemoto-Kohsaka-Takahashi fixed point theorem properly in Section 4. Section 5 is devoting to studying the weak convergence problem for this new class of nonlinear mappings.

\section{Preliminaries}

In what follows, $X$ will be a real Banach space with topological dual $X^{*}$ and $f: X \rightarrow$ $(-\infty, \infty]$ will be a convex function. $\mathcal{D}$ denotes the domain of $f$, that is,

$$
\mathcal{D}=\{x \in X: f(x)<\infty\},
$$

and $\mathcal{D}^{\circ}$ denotes the algebraic interior of $\mathcal{D}$, i.e., the subset of $\mathcal{D}$ consisting of all those points $x \in \mathcal{D}$ such that, for any $y \in X \backslash\{x\}$, there is $z$ in the open segment $(x, y)$ with $[x, z] \subseteq \mathcal{D}$. The topological interior of $\mathcal{D}$, denoted by $\operatorname{Int}(\mathcal{D})$, is contained in $\mathcal{D}^{\circ} . f$ is said to be proper provided that $\mathcal{D} \neq \emptyset$. $f$ is called lower semicontinuous (l.s.c.)

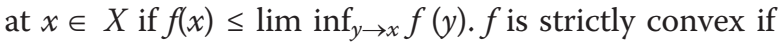

$$
f(\alpha x+(1-\alpha) y)<\alpha f(x)+(1-\alpha) f(\gamma)
$$

for all $x, y \in X$ and $\alpha \in(0,1)$.

The function $f: X \rightarrow(-\infty, \infty]$ is said to be Gâteaux differentiable at $x \in X$ if there is $f(x) \in X^{*}$ such that

$$
\lim _{t \rightarrow 0} \frac{f(x+t y)-f(x)}{t}=\left\langle y, f^{\prime}(x)\right\rangle
$$

for all $y \in X$.

The Bregman distance $D_{f}$ associated with a proper convex function $f$ is the function $D_{f}: \mathcal{D} \times \mathcal{D} \rightarrow[0, \infty]$ defined by 


$$
D_{f}(y, x)=f(y)-f(x)+f^{\circ}(x, x-y)
$$

where $f^{\circ}(x, x-y)=\lim _{t \rightarrow 0^{+}} \frac{f(x+t(x-y))-f(x)}{t}$. $D_{f}(y, x)$ is finite valued if and only if $x \in \mathcal{D}^{\mathrm{o}}$, cf. Proposition 1.1.2 (iv) of [9]. When $f$ is Gâteaux differentiable on $D$, (1) becomes

$$
D_{f}(y, x)=f(y)-f(x)-\left\langle y-x, f^{\prime}(x)\right\rangle,
$$

and then the modulus of total convexity is the function $v_{f}: \mathcal{D}^{\circ} \times[0, \infty) \rightarrow[0, \infty]$ defined by

$$
v_{f}(x, t)=\inf \left\{D_{f}(y, x): y \in \mathcal{D},\|y-x\|=t\right\} .
$$

It is known that

$$
v_{f}(x, c t) \geq c v_{f}(x, t)
$$

for all $t \geq 0$ and $c \geq 1$, cf. Proposition 1.2.2 (ii) of [9]. By definition it follows that

$$
D_{f}(y, x) \geq v_{f}(x,\|y-x\|) .
$$

The modulus of uniform convexity of $f$ is the function $\delta_{f}:[0, \infty) \rightarrow[0, \infty]$ defined by

$$
\delta_{f}(t)=\inf \left\{f(x)+f(y)-2 f\left(\frac{x+y}{2}\right): x, y \in \mathcal{D},\|x-y\| \geq t\right\} .
$$

The function $f$ is called uniformly convex if $\delta_{f}(t)>0$ for all $t>0$. If $f$ is uniformly convex then for any $\varepsilon>0$ there is $\delta>0$ such that

$$
f\left(\frac{x+y}{2}\right) \leq \frac{f(x)}{2}+\frac{f(y)}{2}-\delta
$$

for all $x, y \in \mathcal{D}$ with $\|x-y\| \geq \varepsilon$.

Note that for $y \in \mathcal{D}$ and $x \in \mathcal{D}^{\circ}$, we have

$$
\begin{aligned}
& f(x)+f(y)-2 f\left(\frac{x+y}{2}\right) \\
= & f(y)-f(x)-\frac{f\left(x+\frac{y-x}{2}\right)-f(x)}{\frac{1}{2}} \\
\leq & f(y)-f(x)-f^{\circ}(x, y-x) \leq D_{f}(y, x),
\end{aligned}
$$

where the first inequality follows from the fact that the function $t \rightarrow f(x+t z)-f(x) / t$ is nondecreasing on $(0, \infty)$. Therefore,

$$
v_{f}(x, t) \geq \delta_{f}(t)
$$

whenever $x \in \mathcal{D}^{\circ}$ and $t \geq 0$. For other properties of the Bregman distance $D_{f}$, we refer readers to [9].

The normalized duality mapping $J$ from $X$ to $2^{X^{*}}$ is defined by

$$
J x=\left\{x^{*} \in X^{*}:\left\langle x, x^{*}\right\rangle=\|x\|^{2}=\left\|x^{*}\right\|^{2}\right\}
$$

for all $x \in X$. 
When $f(x)=\|x\|^{2}$ in a smooth Banach space $X$, it is known that $f(x)=2 J(x)$ for $x \in$ $X$, cf. Corollaries 1.2.7 and 1.4.5 of [10]. Hence, we have

$$
\begin{aligned}
D_{f}(y, x) & =\|y\|^{2}-\|x\|^{2}-\left\langle y-x, f^{\prime}(x)\right\rangle \\
& =\|y\|^{2}-\|x\|^{2}-2\langle y-x, J x\rangle \\
& =\|y\|^{2}+\|x\|^{2}-2\langle y, J x\rangle .
\end{aligned}
$$

Moreover, as the normalized duality mapping $J$ in a Hilbert space $H$ is the identity operator, we have

$$
D_{f}(y, x)=\|y\|^{2}+\|x\|^{2}-2\langle y, x\rangle=\|y-x\|^{2} .
$$

Thus, in case $\lambda$ is a constant function and $f(x)=\|x\|^{2}$ in a Hilbert space, (1.5) coincides with (1.4). However, in general, they are different.

A function $g: X \rightarrow(-\infty, \infty]$ is said to be subdifferentiable at a point $x \in X$ if there exists a linear functional $x^{*} \in X^{*}$ such that

$$
g(y)-g(x) \geq\left\langle y-x, x^{*}\right\rangle, \quad \forall y \in X .
$$

We call such $x *$ the subgradient of $g$ at $x$. The set of all subgradients of $g$ at $x$ is denoted by $\partial g(x)$ and the mapping $\partial g: X \rightarrow 2^{X^{*}}$ is called the subdifferential of $g$. For a l.s.c. convex function $f, \partial f$ is bounded on bounded subsets of $\operatorname{Int}(\mathcal{D})$ if and only if $f$ is bounded on bounded subsets there, cf. Proposition 1.1.11 of [9]. A proper convex l.s.c. function $f$ is Gâteaux differentiable at $x \in \operatorname{Int}(\mathcal{D})$ if and only if it has a unique subgradient at $x$; in such case $\partial f(x)=f(x)$, cf. Corollary 1.2.7 of [10].

The following lemma will be quoted in the sequel.

Lemma 2.1. (Proposition 1.1.9 of [9]) If a proper convex function $f: X \rightarrow(-\infty, \infty]$ is Gâteaux differentiable on $\operatorname{Int}(\mathcal{D})$ in a Banach space $X$, then the following statements are equivalent:

(a) The function $f$ is strictly convex on $\operatorname{Int}(\mathcal{D})$.

(b) For any two distinct points $x, y \in \operatorname{Int}(\mathcal{D})$, one has $D_{f}(y, x)>0$.

(c) For any two distinct points $x, y \in \operatorname{Int}(\mathcal{D})$, one has

$$
\left\langle x-y, f^{\prime}(x)-f^{\prime}(y)\right\rangle>0 .
$$

Throughout this article, $F(T)$ will denote the set of all fixed points of a mapping $T$.

\section{Fixed point theorems}

In this section, we apply Lemma 2.1 to study the fixed point problem for mappings satisfying (1.5).

Theorem 3.1. Let $X$ be a reflexive Banach space and let $f: X \rightarrow(-\infty, \infty]$ be a l.s.c. strictly convex function so that it is Gâteaux differentiable on $\operatorname{Int}(\mathcal{D})$ and is bounded on bounded subsets of $\operatorname{Int}(\mathcal{D})$. Suppose $C \subseteq \operatorname{Int}(\mathcal{D})$ is a nonempty closed convex subset of $X$ and $T: C \rightarrow C$ is point-dependent $\lambda$-hybrid relative to $D_{f}$ for some function $\lambda: C \rightarrow \mathbb{R}$. For $x \in C$ and any $n \in \mathbb{N}$ define

$$
S_{n} x=\frac{1}{n} \sum_{k=0}^{n-1} T^{k} x
$$


where $T^{0}$ is the identity mapping on $C$. If $\left\{T^{n} x\right\}_{n \in \mathbb{N}}$ is bounded, then every weak cluster point of $\left\{S_{n} x\right\}_{n \in \mathbb{N}}$ is a fixed point of $T$.

Proof. Since $T$ is point-dependent $\lambda$-hybrid relative to $D_{f}$, we have, for any $y \in C$ and $k \in \mathbb{N} \cup\{0\}$,

$$
\begin{aligned}
0 \leq & D_{f}\left(T^{k} x, y\right)-D_{f}\left(T^{k+1} x, T y\right)+\lambda(y)\left\langle T^{k} x-T^{k+1} x, f^{\prime}(y)-f^{\prime}(T y)\right\rangle \\
= & f\left(T^{k} x\right)-f(y)-\left\langle T^{k} x-y, f^{\prime}(y)\right\rangle-f\left(T^{k+1} x\right)+f(T y)+\left\langle T^{k+1} x-T y, f^{\prime}(T y)\right\rangle \\
& +\lambda(y)\left\langle T^{k} x-T^{k+1} x, f^{\prime}(y)-f^{\prime}(T y)\right\rangle \\
= & {\left[f\left(T^{k} x\right)-f\left(T^{k+1} x\right)\right]+[f(T y)-f(y)]+\left\langle\lambda(y)\left(T^{k} x-T^{k+1} x\right)-T^{k} x+y, f^{\prime}(y)\right\rangle } \\
& +\left\langle T^{k+1} x-T y-\lambda(y)\left(T^{k} x-T^{k+1} x\right), f^{\prime}(T y)\right\rangle .
\end{aligned}
$$

Summing up these inequalities with respect to $k=0,1, \ldots, n-1$, we get

$$
\begin{aligned}
0 \leq & {\left[f(x)-f\left(T^{n} x\right)\right]+n[f(T y)-f(y)]+\left\langle\lambda(y)\left(x-T^{n} x\right)+n y-n S_{n} x, f^{\prime}(y)\right\rangle } \\
& +\left\langle(n+1) S_{n+1} x-x-n T y-\lambda(y)\left(x-T^{n} x\right), f^{\prime}(T y)\right\rangle .
\end{aligned}
$$

Dividing the above inequality by $n$, we have

$$
\begin{aligned}
0 \leq & \frac{f(x)-f\left(T^{n} x\right)}{n}+[f(T y)-f(y)]+\left\langle\frac{\lambda(y)\left(x-T^{n} x\right)}{n}+y-S_{n} x, f^{\prime}(y)\right\rangle \\
& +\left\langle\frac{n+1}{n} S_{n+1} x-\frac{x}{n}-T y-\frac{\lambda(y)\left(x-T^{n} x\right)}{n}, f^{\prime}(T y)\right\rangle .
\end{aligned}
$$

Since $\left\{T^{n} x\right\}_{n \in \mathbb{N}}$ is bounded, $\left\{S_{n} x\right\}_{n \in \mathbb{N}}$ is bounded, and so, in view of $X$ being reflexive, it has a subsequence $\left\{S_{n_{i}} x\right\}_{i \in \mathbb{N}}$ so that $S_{n_{i}} x$ converges weakly to some $v \in C$ as $n_{i} \rightarrow$ $\infty$. Replacing $n$ by $n_{i}$ in (7), and letting $n_{i} \rightarrow \infty$, we obtain from the fact that $\left\{T^{n} x\right\}_{n \in \mathbb{N}}$ and $\left\{f\left(T^{n} x\right)\right\}_{n \in \mathbb{N}}$ are bounded that

$$
0 \leq f(T y)-f(y)+\left\langle y-v, f^{\prime}(y)\right\rangle+\left\langle v-T y, f^{\prime}(T y)\right\rangle .
$$

Putting $y=v$ in (8), we get

$$
0 \leq f(T v)-f(v)+\left\langle v-T v, f^{\prime}(T v)\right\rangle,
$$

that is,

$$
0 \leq-D_{f}(v, T v)
$$

from which follows that $D_{f}(v, T v)=0$. Therefore $T v=v$ by Lemma 2.1. $\square$

The following theorem comes from Theorem 3.1 immediately.

Theorem 3.2. Let $X$ be a reflexive Banach space and let $f: X \rightarrow(-\infty, \infty]$ be a l.s.c. strictly convex function so that it is Gâteaux differentiable on $\operatorname{Int}(\mathcal{D})$ and is bounded on bounded subsets of $\operatorname{Int}(\mathcal{D})$. Suppose $C \subseteq \operatorname{Int}(\mathcal{D})$ is a nonempty closed convex subset of $X$ and $T: C \rightarrow C$ is point-dependent $\lambda$-hybrid relative to $D_{f}$ for some function $\lambda: C \rightarrow \mathbb{R}$. Then, the following two statements are equivalent:

(a) There is a point $x \in C$ such that $\left\{T^{n} x\right\}_{n \in \mathbb{N}}$ is bounded.

(b) $F(T) \neq \varnothing$.

Taking $\lambda(x)=\lambda$, a constant real number, for all $x \in C$ and noting the function $f(x)=$ $\|x\|^{2}$ in a Hilbert space $H$ satisfies all the requirements of Theorem 3.2, the corollary below follows immediately. 
Corollary 3.3. [8] Let $C$ be a nonempty closed convex subset of Hilbert space $H$ and suppose $T: C \rightarrow C$ is $\lambda$-hybrid. Then, the following two statements are equivalent:

(a) There exists $x \in C$ such that $\left\{T^{n}(x)\right\}_{n \in \mathbb{N}}$ is bounded.

(b) $T$ has a fixed point.

We now show that the fixed point set $F(T)$ is closed and convex under the assumptions of Theorem 3.2.

A mapping $T: C \rightarrow X$ is said to be quasi-nonexpansive with respect to $D_{f}$ if $F(T) \neq$ $\varnothing$ and $D_{f}(v, T x) \leq D_{f}(v, x)$ for all $x \in C$ and all $v \in F(T)$.

Lemma 3.4. Let $f: X \rightarrow(-\infty, \infty]$ be a proper strictly convex function on a Banach space $X$ so that it is Gâteaux differentiable on $\operatorname{Int}(\mathcal{D})$, and let $C \subseteq \operatorname{Int}(\mathcal{D})$ be a nonempty closed convex subset of $X$. If $T: C \rightarrow C$ is quasi-nonexpansive with respect to $D_{f}$ then $F(T)$ is a closed convex subset.

Proof. Let $x \in \overline{F(T)}$ and choose $\left\{x_{n}\right\}_{n \in \mathbb{N}} \subseteq F(T)$ such that $x_{n} \rightarrow x$ as $n \rightarrow \infty$. By the continuity of $D_{f}(\cdot, T x)$ and $D_{f}\left(x_{n}, T_{x}\right) \leq D_{f}\left(x_{n}, x\right)$, we have

$$
D_{f}(x, T x)=\lim _{n \rightarrow \infty} D_{f}\left(x_{n}, T x\right) \leq \lim _{n \rightarrow \infty} D_{f}\left(x_{n}, x\right)=D_{f}(x, x)=0 .
$$

Thus, due to the strict convexity of $f$, it follows from Lemma 2.2 that $T x=x$. This shows $F(T)$ is closed. Next, let $x, y \in F(T)$ and $\alpha \in[0,1]$. Put $z=\alpha x+(1-\alpha) y$. We show that $T z=z$ to conclude $F(T)$ is convex. Indeed,

$$
\begin{aligned}
& D_{f}(z, T z) \\
&=f(z)-f(T z)-\left\langle z-T z, f^{\prime}(T z)\right\rangle \\
&=f(z)+[\alpha f(x)+(1-\alpha) f(y)]-f(T z)-\left\langle z-T z, f^{\prime}(T z)\right\rangle-[\alpha f(x)+(1-\alpha) f(y)] \\
&=f(z)+\alpha\left[f(x)-f(T z)-\left\langle x-T z, f^{\prime}(T z)\right\rangle\right] \\
& \quad+(1-\alpha)\left[f(y)-f(T z)-\left\langle y-T z, f^{\prime}(T z)\right\rangle\right]-[\alpha f(x)+(1-\alpha) f(y)] \\
&=f(z)+\alpha D_{f}(x, T z)+(1-\alpha) D_{f}(y, T z)-[\alpha f(x)+(1-\alpha) f(y)] \\
& \leq f(z)+\alpha D_{f}(x, z)+(1-\alpha) D_{f}(y, z)-[\alpha f(x)+(1-\alpha) f(y)] \\
&= f(z)+\alpha\left[f(x)-f(z)-\left\langle x-z, f^{\prime}(z)\right\rangle\right]+(1-\alpha)\left[f(y)-f(z)-\left\langle y-z, f^{\prime}(z)\right\rangle\right] \\
&-[\alpha f(x)+(1-\alpha) f(y)] \\
&= f(z)+\alpha f(x)-\alpha f(z)-\left\langle\alpha x-\alpha z, f^{\prime}(z)\right\rangle+(1-\alpha) f(y)-(1-\alpha) f(z) \\
&-\left\langle(1-\alpha) y-(1-\alpha) z, f^{\prime}(z)\right\rangle-[\alpha f(x)+(1-\alpha) f(y)] \\
&=-\left\langle\alpha x+(1-\alpha) y-(\alpha z+(1-\alpha) z), f^{\prime}(z)\right\rangle \\
&=-\left\langle 0, f^{\prime}(z)\right\rangle=0 .
\end{aligned}
$$

Therefore, $T z=z$ by the strictly convex of $f$. This completes the proof. $\square$

Proposition 3.5. Let $f: X \rightarrow(-\infty, \infty]$ be a proper strictly convex function on a reflexive Banach space $X$ so that it is Gâteaux differentiable on $\operatorname{Int}(\mathcal{D})$ and is bounded on bounded subsets of Int $(D)$, and let $C \subseteq \operatorname{Int}(\mathcal{D})$ be a nonempty closed convex subset of $X$. Suppose $T: C \rightarrow C$ is point-dependent $\lambda$-hybrid relative to $D_{f}$ for some function $\lambda$ : $C \rightarrow \mathbb{R}$ and has a point $x_{0} \in C$ such that $\left\{T^{n}\left(x_{0}\right)\right\}_{n \in \mathbb{N}}$ is bounded. Then, $T$ is quasi-nonexpansive with respect to $D_{f}$ and therefore, $F(T)$ is a nonempty closed convex subset of $C$.

Proof. In view of Theorem 3.2, $F(T) \neq \varnothing$. Now, for any $v \in F(T)$ and any $y \in C$, as $T$ is point-dependent $\lambda$-hybrid relative to $D_{f}$, we have 


$$
\begin{aligned}
D_{f}(v, T y) & =D_{f}(T v, T y) \\
& \leq D_{f}(v, y)+\lambda(y)\left\langle v-T v, f^{\prime}(y)-f^{\prime}(T y)\right\rangle \\
& =D_{f}(v, y)
\end{aligned}
$$

for all $y \in C$, so $T$ is quasi-nonexpansive with respect to $D_{f}$, and hence, $F(T)$ is a nonempty closed convex subset of $C$ by Lemma 3.4. $\square$

For the remainder of this section, we establish a common fixed point theorem for a commutative family of point-dependent $\lambda$-hybrid mappings relative to $D_{f}$.

Lemma 3.6. Let $X$ be a reflexive Banach space and let $f: X \rightarrow(-\infty, \infty]$ be a l.s.c. strictly convex function so that it is Gâteaux differentiable on $\operatorname{Int}(\mathcal{D})$ and is bounded on bounded subsets of $\operatorname{Int}(\mathcal{D})$. Suppose $C \subseteq \operatorname{Int}(\mathcal{D})$ is a nonempty bounded closed convex subset of $X$ and $\left\{T_{1}, T_{2}, \ldots, T_{N}\right\}$ is a commutative finite family of point-dependent $\lambda$ hybrid mappings relative to $D_{f}$ for some function $\lambda: C \rightarrow \mathbb{R}$ from $C$ into itself. Then $\left\{T_{1}, T_{2}, \ldots, T_{N}\right\}$ has a common fixed point.

Proof. We prove this lemma by induction with respect to $N$. To begin with, we deal with the case that $N=2$. By Proposition 3.5, we see that $F\left(T_{1}\right)$ and $F\left(T_{2}\right)$ are nonempty bounded closed convex subsets of $X$. Moreover, $F\left(T_{1}\right)$ is $T_{2}$-invariant. Indeed, for any $v$ $\in F\left(T_{1}\right)$, it follows from $T_{1} T_{2}=T_{2} T_{1}$ that $T_{1} T_{2} v=T_{2} T_{1} v=T_{2} v$, which shows that $T_{2} v$ $\in F\left(T_{1}\right)$. Consequently, the restriction of $T_{2}$ to $F\left(T_{1}\right)$ is point-dependent $\lambda$-hybrid relative to $D_{f}$, and hence by Theorem 3.2, $T_{2}$ has a fixed point $u \in F\left(T_{1}\right)$, that is, $u \in F$ $\left(T_{1}\right) \cap F\left(T_{2}\right)$.

By induction hypothesis, assume that for some $n \geq 2, E=\cap_{k=1}^{n} F\left(T_{k}\right)$ is nonempty. Then, $E$ is a nonempty closed convex subset of $X$ and the restriction of $T_{n+1}$ to $E$ is a point-dependent $\lambda$-hybrid mapping relative to $D_{f}$ from $E$ into itself. By Theorem 3.2, $T_{n+1}$ has a fixed point in $X$. This shows that $E \cap F\left(T_{n+1}\right) \neq \varnothing$, that is, $\cap_{k=1}^{n+1} F\left(T_{k}\right) \neq \emptyset$, completing the proof. $\square$.

Theorem 3.7. Let $X$ be a reflexive Banach space and let $f: X \rightarrow(-\infty, \infty]$ be a l.s.c. strictly convex function so that it is Gâteaux differentiable on $\operatorname{Int}(\mathcal{D})$. Suppose $C \subseteq \operatorname{Int}(\mathcal{D})$ is a nonempty bounded closed convex subset of $X$ and $\left\{T_{i}\right\}_{i L}$ is a commutative family of pointdependent $\lambda$-hybrid mappings relative to $D_{f}$ for some function $\lambda: C \rightarrow \mathbb{R}$ from $C$ into itself. Then, $\left\{T_{i}\right\}_{\text {il }}$ has a common fixed point.

Proof. Since $C$ is a nonempty bounded closed convex subset of the reflexive Banach space $X$, it is weakly compact. By Proposition 3.5, each $F\left(T_{i}\right)$ is a nonempty weakly compact subset of $C$. Therefore, the conclusion follows once we note that $\left\{F\left(T_{i}\right)\right\}_{i L I}$ has the finite intersection property by Lemma 3.6. $\square$.

\section{Examples}

In this section, we give some concrete examples for our fixed point theorem. At first, we need a lemma.

Lemma 4.1. Let $h$ and $k$ be two real numbers in $[0,1]$. Then, the following two statements are true.

(a) $\left(h^{2}-k^{2}\right)^{2}-(h-k)^{2} \geq 0$, if $\frac{h+k}{2}>0.5$.

(b) $\left(h^{2}-k^{2}\right)^{2}-(h-k)^{2} \leq 0$, if $\frac{h+k}{2} \leq 0.5$. 
Proof. First, we represent $h$ and $k$ by

$$
h=0.5+a, \quad \text { where }-0.5 \leq a \leq 0.5,
$$

and

$$
k=0.5+b, \quad \text { where }-0.5 \leq b \leq 0.5 .
$$

Then, we have

$$
\left(h^{2}-k^{2}\right)^{2}-(h-k)^{2}=(a-b)^{2}(a+b)(a+b+2) .
$$

If $\frac{h+k}{2}>0.5$, then $a+b>0$, and so through the above equation, we obtain that $\left(h^{2}-\right.$ $\left.k^{2}\right)^{2}-(h-k)^{2} \geq 0$. On the other hand, $\frac{h+k}{2} \leq 0.5$ implies $a+b \leq 0$, and hence, $\left(h^{2}\right.$ $\left.k^{2}\right)^{2}-(h-k)^{2} \leq 0$.

Example 4.2. Let $C=\left\{x \in l^{2}(\mathbb{N}): x=\left(x_{1}, x_{2}, \ldots, x_{n}, \ldots\right), 0 \leq x_{i} \leq 1-\frac{1}{i+1}\right\}$ and $\delta$ be a positive number so small that $\sqrt{\delta}<0.5$. Define a mapping $T: C \rightarrow C$ by

$$
T x=\left(T x_{1}, T x_{2}, \ldots, T x_{n}, \ldots\right): T x_{i}=\left\{\begin{array}{l}
x_{i}^{2}, \text { if } \sqrt{\delta}<x_{i} \leq 1-\frac{1}{i+1} \\
\delta, \text { if } \delta<x_{i} \leq \sqrt{\delta} \\
x_{i}, \text { if } 0 \leq x_{i} \leq \delta .
\end{array}\right.
$$

Then for any $\lambda \in \mathbb{R}, T$ is not $\lambda$-hybrid. However, for each $x \in C$, if we let $n_{x}=\min \left\{n: \sum_{i=n+1}^{\infty} x_{i}^{2} \leq \delta^{2}\right\}$ and define $\lambda: C \rightarrow \mathbb{R}$ by

$$
\lambda(x)=\frac{1}{\left(\frac{1}{n_{x}+1}-\frac{1}{\left(n_{x}+1\right)^{2}}\right)^{2}}
$$

then $T$ is point-dependent $\lambda$-hybrid, that is,

$$
\|T x-T y\|^{2} \leq\|x-y\|^{2}+\lambda(y)\langle x-T x, y-T y\rangle
$$

for all $x, y \in C$. Therefore, we can apply Theorem 3.2 to conclude that $T$ has a fixed point, while the Aoyama-Iemoto-Kohsaka-Takahashi fixed point theorem fails to give us the desired conclusion.

Proof. Let $x$ and $y$ be two elements from $C$ so that the $m^{\text {th }}$ coordinate of $x$ is $1-\frac{1}{m+1}$ the $m^{\text {th }}$ coordinate of $y$ is 0.5 and the rest coordinates of $x$ and $y$ are zero. We have

$$
\begin{aligned}
& \|T x-T y\|^{2}-\|x-y\|^{2}-m\langle x-T x, y-T y\rangle \\
= & {\left[\left(1-\frac{1}{m+1}\right)^{2}-(0.5)^{2}\right]^{2}-\left[\left(1-\frac{1}{m+1}\right)-0.5\right]^{2} } \\
& -m\left[\left(1-\frac{1}{m+1}\right)-\left(1-\frac{1}{m+1}\right)^{2}\right]\left[0.5-(0.5)^{2}\right] \\
= & \frac{9}{16}-\frac{2}{m+1}+\frac{9}{2(m+1)^{2}}-\frac{4}{(m+1)^{3}}+\frac{1}{(m+1)^{4}}-\frac{m^{2}}{4(m+1)^{2}} \\
& \rightarrow \frac{5}{16} \text { as } m \rightarrow \infty .
\end{aligned}
$$

Since the value of above equality is always positive as $m$ is large enough, we conclude that there is no constant $\lambda$ to satisfy the inequality: 


$$
\|T x-T y\|^{2} \leq\|x-y\|^{2}+\lambda\langle x-T x, y-T y\rangle
$$

for all $x, y \in C$.

It remains to show that $T$ satisfies the inequality (9). We can rewrite the inequality as

$$
\sum_{i=1}^{\infty}\left(T x_{i}-T y_{i}\right)^{2} \leq \sum_{i=1}^{\infty}\left(x_{i}-y_{i}\right)^{2}+\sum_{i=1}^{\infty} \lambda(y)\left(x_{i}-T x_{i}\right)\left(y_{i}-T y_{i}\right)
$$

Thus, if we can show that for all $i \in \mathbb{N}$,

$$
\left(T x_{i}-T y_{i}\right)^{2} \leq\left(x_{i}-y_{i}\right)^{2}+\lambda(y)\left(x_{i}-T x_{i}\right)\left(y_{i}-T y_{i}\right)
$$

then the assertion follows. We prove inequality (10) holds for all $i \in \mathbb{N}$ by considering the following two cases: (I) $i>\min \left\{n_{x}, n_{y}\right\}$ and (II) $i \leq \min \left\{n_{x}, n_{y}\right\}$.

- Case (I). $i>\min \left\{n_{x}, n_{y}\right\}$.

In this case, at least one of $x_{i}$ and $y_{i}$ is less than or equal to $\delta$. Suppose that $0 \leq x_{i} \leq$ $\delta$. There are three subcases to discuss.

(I-1): If $\sqrt{\delta}<y_{i} \leq 1-\frac{1}{i+1}$, then we have

$$
\begin{aligned}
\left(T x_{i}-T y_{i}\right)^{2}=\left(x_{i}-y_{i}^{2}\right)^{2} & \leq\left(x_{i}-y_{i}\right)^{2} \\
& \leq\left(x_{i}-y_{i}\right)^{2}+\lambda(y)\left(x_{i}-T x_{i}\right)\left(y_{i}-T y_{i}\right)
\end{aligned}
$$

$(\mathrm{I}-2): \delta<y_{i} \leq \sqrt{\delta}$, then we have

$$
\begin{aligned}
\left(T x_{i}-T y_{i}\right)^{2}=\left(x_{i}-\delta\right)^{2} & \leq\left(x_{i}-y_{i}\right)^{2} \\
& \leq\left(x_{i}-y_{i}\right)^{2}+\lambda(y)\left(x_{i}-T x_{i}\right)\left(y_{i}-T y_{i}\right)
\end{aligned}
$$

(I-3): If $0 \leq y_{i} \leq \delta$, then we have

$$
\left(T x_{i}-T y_{i}\right)^{2}=\left(x_{i}-y_{i}\right)^{2} \leq\left(x_{i}-y_{i}\right)^{2}+\lambda(y)\left(x_{i}-T x_{i}\right)\left(y_{i}-T y_{i}\right)
$$

The case that $0 \leq y_{i} \leq \delta$ can be proved in the same manner.

- Case (II). $i \leq \min \left\{n_{x}, n_{y}\right\}$.

In this case, there are 9 subcases to discuss.

(II-1): $\sqrt{\delta}<x_{i} \leq 1-\frac{1}{i+1}$ and $\sqrt{\delta}<y_{i} \leq 1-\frac{1}{i+1}$.

If $\frac{x_{i}+y_{i}}{2} \leq 0.5$, it follows from Lemma 4.1 that

$$
\begin{aligned}
\left(T x_{i}-T y_{i}\right)^{2}=\left(x_{i}^{2}-y_{i}^{2}\right)^{2} & \leq\left(x_{i}-y_{i}\right)^{2} \\
& \leq\left(x_{i}-y_{i}\right)^{2}+\lambda(y)\left(x_{i}-T x_{i}\right)\left(y_{i}-T y_{i}\right)
\end{aligned}
$$

If $\frac{x_{i}+y_{i}}{2}>0.5$, then both $x_{i}$ and $y_{i}$ are greater than $\frac{1}{i+1}$, and so by considering the graph of the function $g(z)=z-z^{2}$ in $\mathbb{R}$, which is symmetric to the line $L: x=0.5$, we have

$$
\left(x_{i}-x_{i}^{2}\right) \geq\left(\frac{1}{i+1}\right)-\left(\frac{1}{i+1}\right)^{2} \geq\left(\frac{1}{n_{y}+1}\right)-\left(\frac{1}{n_{y}+1}\right)^{2}
$$

and

$$
\left(y_{i}-y_{i}^{2}\right) \geq\left(\frac{1}{i+1}\right)-\left(\frac{1}{i+1}\right)^{2} \geq\left(\frac{1}{n_{y}+1}\right)-\left(\frac{1}{n_{y}+1}\right)^{2} .
$$


Consequently, we obtain

$$
\begin{aligned}
\left(T x_{i}-T y_{i}\right)^{2}=\left(x_{i}^{2}-y_{i}^{2}\right)^{2} \leq 1 & \leq \frac{1}{\left(\frac{1}{n_{y}+1}-\frac{1}{\left(n_{y}+1\right)^{2}}\right)^{2}}\left(x_{i}-x_{i}^{2}\right)\left(y_{i}-y_{i}^{2}\right) \\
& \leq\left(x_{i}-y_{i}\right)^{2}+\lambda(y)\left(x_{i}-T x_{i}\right)\left(y_{i}-T y_{i}\right) .
\end{aligned}
$$

(II-2): $\delta<x_{i} \leq \sqrt{\delta}$ and $\sqrt{\delta}<y_{i} \leq 1-\frac{1}{i+1}$.

If $y_{i} \leq 0.5$, then $\frac{x_{i}+y_{i}}{2}<0.5$. Thus, from Lemma 4.1, we have

$$
\begin{aligned}
\left(T x_{i}-T y_{i}\right)^{2}=\left(\delta-y_{i}^{2}\right)^{2} & \leq\left(x_{i}^{2}-y_{i}^{2}\right)^{2} \\
& \leq\left(x_{i}-y_{i}\right)^{2} \\
& \leq\left(x_{i}-y_{i}\right)^{2}+\lambda(y)\left(x_{i}-T x_{i}\right)\left(y_{i}-T y_{i}\right) .
\end{aligned}
$$

If $y_{i}>0.5$, we have either

$$
\delta<x_{i} \leq \delta+\left(\frac{1}{i+1}\right)-\left(\frac{1}{i+1}\right)^{2}
$$

or

$$
\delta+\left(\frac{1}{i+1}\right)-\left(\frac{1}{i+1}\right)^{2}<x_{i} \leq \sqrt{\delta} .
$$

When $\delta<x_{i} \leq \delta+\left(\frac{1}{i+1}\right)-\left(\frac{1}{i+1}\right)^{2}$, by considering the graph of the function $g(z)=z-$ $z^{2}$ in $\mathbb{R}$, we have

$$
y_{i}-y_{i}^{2} \geq\left(\frac{1}{i+1}\right)-\left(\frac{1}{i+1}\right)^{2} \geq x_{i}-\delta .
$$

and thus, we obtain

$$
y_{i}-x_{i} \geq y_{i}^{2}-\delta>0 .
$$

Therefore,

$$
\begin{aligned}
\left(T x_{i}-T y_{i}\right)^{2} & =\left(\delta-y_{i}^{2}\right)^{2} \\
& \leq\left(x_{i}-y_{i}\right)^{2} \leq\left(x_{i}-y_{i}\right)^{2}+\lambda(y)\left(x_{i}-T x_{i}\right)\left(y_{i}-T y_{i}\right) .
\end{aligned}
$$

When $\delta+\left(\frac{1}{i+1}\right)-\left(\frac{1}{i+1}\right)^{2}<x_{i} \leq \sqrt{\delta}$, both of $x_{i}-\delta$ and $y_{i}-y_{i}^{2}$ are greater than $\left(\frac{1}{i+1}\right)-\left(\frac{1}{i+1}\right)^{2}$ and thus also greater than $\left(\frac{1}{n_{y}+1}\right)-\left(\frac{1}{n_{y}+1}\right)^{2}$.

Therefore,

$$
\begin{aligned}
\left(T x_{i}-T y_{i}\right)^{2}=\left(\delta-y_{i}^{2}\right)^{2} \leq 1 & \leq \frac{1}{\left(\frac{1}{n_{y}+1}-\frac{1}{\left(n_{y}+1\right)^{2}}\right)^{2}}\left(x_{i}-\delta\right)\left(y_{i}-y_{i}^{2}\right) \\
& \leq\left(x_{i}-y_{i}\right)^{2}+\lambda(y)\left(x_{i}-T x_{i}\right)\left(y_{i}-T y_{i}\right) .
\end{aligned}
$$

Likely, we can prove the case:

(II-3): $\sqrt{\delta}<x_{i} \leq 1-\frac{1}{i+1}$ and $\delta<y_{i} \leq \sqrt{\delta}$.

(II-4): $0 \leq x_{i} \leq \delta$ and $\sqrt{\delta}<y_{i} \leq 1-\frac{1}{i+1}$. 
Then, we have

$$
\begin{aligned}
\left(T x_{i}-T y_{i}\right)^{2}=\left(x_{i}-y_{i}^{2}\right)^{2} & \leq\left(x_{i}-y_{i}\right)^{2} \\
& \leq\left(x_{i}-y_{i}\right)^{2}+\lambda(y)\left(x_{i}-T x_{i}\right)\left(y_{i}-T y_{i}\right)
\end{aligned}
$$

Similarly, we can prove the case:

(II-5): $\sqrt{\delta}<x_{i} \leq 1-\frac{1}{i+1}$ and $0 \leq y_{i} \leq \delta$.

(II-6): $\delta<x_{i} \leq \sqrt{\delta}$ and $\delta<y_{i} \leq \sqrt{\delta}$.

In this case, we have

$$
\left(T x_{i}-T y_{i}\right)^{2}=(\delta-\delta)^{2}=0 \leq\left(x_{i}-y_{i}\right)^{2}+\lambda(y)\left(x_{i}-T x_{i}\right)\left(y_{i}-T y_{i}\right)
$$

(II-7): $0 \leq x_{i} \leq \delta$ and $\delta<y_{i} \leq \sqrt{\delta}$.

This case can be treated as (I-2).

(II-8): $0 \leq x_{i} \leq \delta$ and $0 \leq y_{i} \leq \delta$.

This case can be treated as (I-3).

(II-9): $\delta<x_{i} \leq \sqrt{\delta}$ and $0 \leq y_{i} \leq \delta$.

This case can be treated as (I-2). $\square$

To end this section, we give another example which shows that the concept of a nonspreading mapping in the sense of (1.2) is generally different from that of a 2hybrid mapping relative to some $D_{f}$ in Hilbert spaces.

Example 4.3. Define $f: \mathbb{R} \rightarrow \mathbb{R}$ by $f(x)=x^{10}$ for all $x \in \mathbb{R}$, and define $T:[0,0.85] \rightarrow$ $[0,0.85]$ by $T x=x^{2}$ for all $x \in[0,0.85]$. Then, $T$ is neither nonexpansive nor nonspreading, but it is $\lambda$-hybrid relative to $D_{f}$ for any $\lambda \geq 0$. Thus, we can apply Theorem 3.2 to conclude $T$ has a fixed point, while both of the Browder Fixed Point Theorem and the Kohsaka-Takahashi fixed point theorem fail.

Proof. It is easy to check that $T$ is not nonexpansive. As for not nonspreading, taking $x=0.85$ and $y=0.5$, we have

$$
\|T x-T y\|^{2}=\left(x^{2}-y^{2}\right)^{2}=\left[(0.85)^{2}-(0.5)^{2}\right]^{2}=0.22325625
$$

while

$$
\begin{aligned}
& \|x-y\|^{2}+2\langle x-T x, y-T y\rangle \\
= & (x-y)^{2}+2\left(x-x^{2}\right)\left(y-y^{2}\right) \\
= & (0.85-0.5)^{2}+2\left[0.85-(0.85)^{2}\right]\left[0.5-(0.5)^{2}\right]=0.18625
\end{aligned}
$$

Hence, $T$ is not nonspreading in the sense of (1.2). It remains to show that for any $\lambda$ $\geq 0, T$ is $\lambda$-hybrid relative to $D_{f}$. Note at first that, for all $\lambda \geq 0$ and for all $x, y \in[0$, $0.85]$,

$$
\begin{gathered}
\lambda\left\langle x-T x, f^{\prime}(y)-f^{\prime}(T y)\right\rangle \\
=\lambda\left(x-x^{2}\right)\left(10 y^{9}-10 y^{18}\right) \geq 0 .
\end{gathered}
$$

Hence, it suffices to prove that $T$ is 0 -hybrid relative to $D_{f}$, that is, to show that

$$
D_{f}(T x, T y)-D_{f}(x, y) \leq 0, \quad \forall x, y \in[0,0.85] .
$$


Fixed any $x \in[0,0.85]$, let $h(y)=D_{f}\left(T_{x}, T_{y}\right)-D_{f}(x, y)$. Then

$$
\begin{aligned}
h(y) & =f(T x)-f(T y)-\left\langle T x-T y, f^{\prime}(T y)\right\rangle-\left[f(x)-f(y)-\left\langle x-y, f^{\prime}(y)\right\rangle\right] \\
& =x^{20}+9 y^{20}-10 x^{2} y^{18}-x^{10}-9 y^{10}+10 x y^{9} .
\end{aligned}
$$

We have

$$
\begin{aligned}
h^{\prime}(y) & =180 y^{19}-180 x^{2} y^{17}-90 y^{9}+90 x y^{8} \\
& =90 y^{8}\left(2 y^{11}-2 x^{2} y^{9}-y+x\right) \\
& =90 y^{8}\left[2 y^{9}\left(y^{2}-x^{2}\right)-(y-x)\right] \\
& =90 y^{8}\left[2 y^{9}(y+x)(y-x)-(y-x)\right] \\
& =90 y^{8}(y-x)\left[2 y^{9}(y+x)-1\right] .
\end{aligned}
$$

Since $y$ and $x$ are in $[0,0.85]$, one has

$$
2 y^{9}(y+x)-1<2(0.85)^{9}(0.85+0.85)-1<0,
$$

and hence

$$
h^{\prime}(y) \begin{cases}\geq 0, \text { if } y \leq x \\ \leq 0, \text { if } y>x\end{cases}
$$

Moreover, we know $h(y)=0$ if $x=y$. Therefore, $h(y)$ is always less than or equal to zero and we have proved that $D_{f}(T x, T y)-D_{f}(x, y) \leq 0$ for all $x, y \in[0,0.85]$.

\section{Weak convergence theorems}

In this section, we discuss the demiclosedness and the weak convergence problem of point-dependent $\lambda$-hybrid relative to $D_{f}$. We denote the weak convergence and strong convergence of a sequence $\left\{x_{n}\right\}$ to $v$ in a Banach space by $x_{n} \rightarrow v$ and $x_{n} \rightarrow v$, respectively. For a nonempty closed convex subset $C$ of a Banach space $X$, a mapping $T: C$ $\rightarrow X$ is demiclosed if for any sequence $\left\{x_{n}\right\}$ in $C$ with $x_{n} \rightarrow v$ and $x_{n}-T x_{n} \rightarrow 0$, one has $T v=v$.

We first derive an Opial-like inequality for the Bregman distance. For the Opial's inequality, we refer readers to Lemma 1 of [11].

Lemma 5.1. Suppose $f: X \rightarrow(-\infty, \infty]$ is a proper strictly convex function so that it is Gâteaux differentiable on $\operatorname{Int}(\mathcal{D})$ in a Banach space $X$ and $\left\{x_{n}\right\}_{n \in \mathbb{N}}$ is a sequence in $\mathcal{D}$ such that $x_{n} \rightarrow v$ for some $v \in \operatorname{Int}(\mathcal{D})$. Then

$$
\liminf _{n \rightarrow \infty} D_{f}\left(x_{n}, v\right)<\liminf _{n \rightarrow \infty} D_{f}\left(x_{n}, y\right), \quad \forall y \in \operatorname{Int}(\mathcal{D}) \text { with } y \neq v .
$$

Proof. Since

$$
\begin{aligned}
& D_{f}\left(x_{n}, v\right)-D_{f}\left(x_{n}, y\right) \\
= & f\left(x_{n}\right)-f(v)-\left\langle x_{n}-v, f^{\prime}(v)\right\rangle-\left[f\left(x_{n}\right)-f(y)-\left\langle x_{n}-y, f^{\prime}(y)\right\rangle\right] \\
= & \left.f\left(x_{n}\right)-f(v)-\left\langle x_{n}-v, f^{\prime}(v)\right\rangle-f\left(x_{n}\right)+f(y)+\left\langle x_{n}-\gamma, f^{\prime}(y)\right\rangle\right] \\
& +\left\langle x_{n}-v, f^{\prime}(y)\right\rangle-\left\langle x_{n}-v, f^{\prime}(y)\right\rangle \\
= & -\left[f(v)-f(y)-\left\langle v-y, f^{\prime}(y)\right\rangle\right]+\left\langle x_{n}-v, f^{\prime}(y)-f^{\prime}(v)\right\rangle \\
= & -D_{f}(v, y)+\left\langle x_{n}-v, f^{\prime}(y)-f^{\prime}(v)\right\rangle
\end{aligned}
$$


and $x_{n} \rightarrow v$, we have

$$
\lim _{n \rightarrow \infty}\left[D_{f}\left(x_{n}, v\right)-D_{f}\left(x_{n}, y\right)\right]=-D_{f}(v, y) .
$$

Consequently,

$$
\begin{aligned}
\liminf _{n \rightarrow \infty} D_{f}\left(x_{n}, v\right) & =\liminf _{n \rightarrow \infty}\left[\left(D_{f}\left(x_{n}, v\right)-D_{f}\left(x_{n}, y\right)\right)+D_{f}\left(x_{n}, y\right)\right] \\
& =\lim _{n \rightarrow \infty}\left(D_{f}\left(x_{n}, v\right)-D_{f}\left(x_{n}, y\right)\right)+\liminf _{n \rightarrow \infty} D_{f}\left(x_{n}, y\right) \\
& =-D_{f}(v, y)+\liminf _{n \rightarrow \infty} D_{f}\left(x_{n}, y\right),
\end{aligned}
$$

and hence in view of $D_{f}(v, y)>0$ for $y \neq v$ we obtain

$$
\liminf _{n \rightarrow \infty} D_{f}\left(x_{n}, v\right)<\liminf _{n \rightarrow \infty} D_{f}\left(x_{n}, y\right) .
$$

$\square$

Proposition 5.2. Let $f: X \rightarrow(-\infty, \infty]$ be a strictly convex function so that it is Gâteaux differentiable on $\operatorname{Int}(\mathcal{D})$ and is bounded on bounded subsets of $\operatorname{Int}(\mathcal{D})$. Suppose $C$ is a closed convex subset of $\operatorname{Int}(\mathcal{D})$ and $T: C \rightarrow C$ is point-dependent $\lambda$-hybrid relative to $D_{f}$ for some $\lambda: C \rightarrow \mathbb{R}$. Then $T$ is demiclosed.

Proof. Let $\left\{x_{n}\right\}$ be any sequence in $C$ with $x_{n} \rightarrow v$ and $x_{n}-T x_{n} \rightarrow 0$. We have to show that $T v=v$. Since $f$ is bounded on bounded subsets, by Proposition 1.1.11 of [9] there exists a constant $M>0$ such that

$$
\max \left\{\sup \left\{\left\|f^{\prime}\left(x_{n}\right)\right\|: n \in \mathbb{N}\right\},\|\lambda(v)\|,\left\|f^{\prime}(T v)\right\|,\left\|f^{\prime}(v)\right\|\right\} \leq M .
$$

Rewrite $D_{f}\left(x_{n}, T v\right)$ as

$$
\begin{aligned}
D_{f}\left(x_{n}, T v\right)= & f\left(x_{n}\right)-f(T v)-\left\langle x_{n}-T v, f^{\prime}(T v)\right\rangle \\
= & f\left(x_{n}\right)+f\left(T x_{n}\right)-f\left(T x_{n}\right)-f(T v)-\left\langle x_{n}-T v, f^{\prime}(T v)\right\rangle \\
& +\left\langle T x_{n}-T v, f^{\prime}(T v)\right\rangle-\left\langle T x_{n}-T v, f^{\prime}(T v)\right\rangle \\
= & {\left[f\left(T x_{n}\right)-f(T v)-\left\langle T x_{n}-T v, f^{\prime}(T v)\right\rangle\right]+f\left(x_{n}\right)-f\left(T x_{n}\right) } \\
& +\left\langle T x_{n}-x_{n}, f^{\prime}(T v)\right\rangle \\
= & D_{f}\left(T x_{n}, T v\right)+f\left(x_{n}\right)-f\left(T x_{n}\right)+\left\langle T x_{n}-x_{n}, f^{\prime}(T v)\right\rangle .
\end{aligned}
$$

Noting $f\left(x_{n}\right)-f\left(T x_{n}\right) \leq\left\langle x_{n}-T x_{n}, f\left(x_{n}\right)\right\rangle$ and $T$ is point-dependent $\lambda$-hybrid relative to $D_{f}$, we have from (11) that

$$
\begin{aligned}
& D_{f}\left(x_{n}, T v\right) \\
\leq & D_{f}\left(T x_{n}, T v\right)+\left\langle x_{n}-T x_{n}, f^{\prime}\left(x_{n}\right)\right\rangle-\left\langle x_{n}-T x_{n}, f^{\prime}(T v)\right\rangle \\
\leq & D_{f}\left(x_{n}, v\right)+\lambda(v)\left\langle x_{n}-T x_{n}, f^{\prime}(v)-f^{\prime}(T v)\right\rangle+\left\langle x_{n}-T x_{n}, f^{\prime}\left(x_{n}\right)-f^{\prime}(T v)\right\rangle \\
\leq & D_{f}\left(x_{n}, v\right)+\left[|\lambda(v)|\left(\left\|f^{\prime}(v)\right\|+\left\|f^{\prime}(T v)\right\|\right)+\left(\left\|f^{\prime}\left(x_{n}\right)\right\|+\left\|f^{\prime}(T v)\right\|\right)\right]\left\|x_{n}-T x_{n}\right\| \\
\leq & D_{f}\left(x_{n}, v\right)+2 M(M+1)\left\|x_{n}-T x_{n}\right\| .
\end{aligned}
$$

If $T v \neq v$, then Lemma 5.1 and (12) imply that

$$
\begin{aligned}
& \liminf _{n \rightarrow \infty} D_{f}\left(x_{n}, v\right) \\
< & \liminf _{n \rightarrow \infty} D_{f}\left(x_{n}, T v\right) \\
\leq & \liminf _{n \rightarrow \infty}\left[D_{f}\left(x_{n}, v\right)+2 M(M+1)\left\|x_{n}-T x_{n}\right\|\right]=\liminf _{n \rightarrow \infty} D_{f}\left(x_{n}, v\right),
\end{aligned}
$$


a contradiction. This completes the proof. $\square$

A mapping $T: C \rightarrow C$ is said to be asymptotically regular if, for any $x \in C$, the sequence $\left\{T^{n+1} x-T^{n} x\right\}$ tends to zero as $n \rightarrow \infty$.

Theorem 5.3. Suppose the following conditions hold:

(5.3.1) $f: X \rightarrow(-\infty, \infty]$ is l.s.c. uniformly convex function so that it is Gâteaux differentiable on $\operatorname{Int}(\mathcal{D})$ and is bounded on bounded subsets of $\operatorname{Int}(\mathcal{D})$ in a reflexive Banach space $X$.

(5.3.2) $C \subseteq \operatorname{Int}(\mathcal{D})$ is a closed convex subset of $X$.

(5.3.3) $T: C \rightarrow C$ is point-dependent $\lambda$-hybrid relative to $D_{f}$ for some $\lambda: C \rightarrow \mathbb{R}$ and is asymptotically regular with a bounded sequence $\left\{T^{n} x_{0}\right\}_{n \in \mathbb{N}}$ for some $x_{0} \in C$.

(5.3.4) The mapping $x \rightarrow f(x)$ for $x \in X$ is weak-to-weak" continuous.

Then for any $x \in C,\left\{T^{n} x\right\}_{n \in \mathbb{N}}$ is weakly convergent to an element $v \in F(T)$.

Proof. Let $v \in F(T)$ and $x \in C$. If $\left\{T^{n} x\right\}_{n \in \mathbb{N}}$ is not bounded, then there is a subsequence $\left\{T^{n_{i}} x\right\}_{i \in \mathbb{N}}$ such that $\left\|v-T^{n_{i}} x\right\| \geq 1$ for all $i \in \mathbb{N}$ and $\left\|v-T^{n_{i}} x\right\| \rightarrow \infty$ as $i \rightarrow$ $\infty$. From (5.3.3), for any $n \in \mathbb{N}$, we have

$$
\begin{aligned}
D_{f}\left(v, T^{n+1} x\right) & =D_{f}\left(T v, T^{n+1} x\right) \\
& \leq D_{f}\left(v, T^{n} x\right)+\lambda\left(T^{n} x\right)\left\{v-T v, f^{\prime}\left(T^{n} x\right)-f^{\prime}\left(T^{n+1} x\right)\right\}=D_{f}\left(v, T^{n} x\right) \\
& \leq D_{f}(v, x),
\end{aligned}
$$

which in conjunction with (3), (4), and (6) implies that

$$
\begin{aligned}
D_{f}(v, x) & \geq D_{f}\left(v, T^{n_{i}} x\right) \geq v_{f}\left(T^{n_{i}} x,\left\|v-T^{n_{i}} x\right\|\right) \\
& \geq\left\|v-T^{n_{i}} x\right\| v_{f}\left(T^{n_{i}} x, 1\right) \\
& \geq\left\|v-T^{n_{i}} x\right\| \delta_{f}(1) \rightarrow \infty, \quad \text { as } i \rightarrow \infty,
\end{aligned}
$$

a contradiction. Therefore, for any $x \in X,\left\{T^{n} x\right\}_{n \in \mathbb{N}}$ is bounded, and so it has a subsequence $\left\{T^{n_{j}} x\right\}_{j \in \mathbb{N}}$ which is weakly convergent to $w$ for some $w \in C$. As $T^{n_{j}} x-T^{n_{j}+1} x \rightarrow 0$, it follows from the demiclosedness of $T$ that $w \in F(T)$. It remains to show that $T^{n} x-w$ as $n \rightarrow \infty$. Let $\left\{T^{n_{k}} x\right\}_{n \in \mathbb{N}}$ be any subsequence of $\left\{T^{n} x\right\}_{n \in \mathbb{N}}$ so that $T^{n_{k}} x \rightarrow u$ for some $u \in C$. Then $u \in F(T)$. Since both of $\left\{D_{f}\left(w, T^{n} x\right)\right\}_{n \in \mathbb{N}}$ and $\left\{D_{f}\right.$ $\left.\left(u, T^{n} x\right)\right\}_{n \in \mathbb{N}}$ are decreasing, we have

$$
\lim _{n \rightarrow \infty}\left[D_{f}\left(w, T^{n} x\right)-D_{f}\left(u, T^{n} x\right)\right]=\lim _{n \rightarrow \infty}\left[f(w)-f(u)-\left\langle w-u, f^{\prime}\left(T^{n} x\right)\right\rangle\right]=a
$$

for some $a \in \mathbb{R}$. Particularly, from (5.3.4) we obtain

$$
a=\lim _{n_{j} \rightarrow \infty}\left[f(w)-f(u)-\left\langle w-u, f^{\prime}\left(T^{n_{j}} x\right)\right\rangle\right]=f(w)-f(u)-\left\langle w-u, f^{\prime}(w)\right\rangle
$$

and

$$
a=\lim _{n_{k} \rightarrow \infty}\left[f(w)-f(u)-\left\langle w-u, f^{\prime}\left(T^{n_{k}} x\right)\right\rangle\right]=f(w)-f(u)-\left\langle w-u, f^{\prime}(u)\right\rangle .
$$

Consequently, $\langle w-u, f(w)-f(u)\rangle=0$, and hence $w=u$ by the strict convexity of $f$. This shows that $T^{n} x \rightarrow w$ for some $w \in F(T)$.

Adopting the technique of [8], we have the following ergodic theorem for pointdependent $\lambda$-hybrid mappings in Hilbert spaces. 


\section{Theorem 5.4. Suppose}

(5.4.1) $C$ is nonempty closed convex subset of a Hilbert space $H$.

(5.4.2) $T: C \rightarrow C$ is a point-dependent $\lambda$-hybrid mapping for some function $\lambda: C \rightarrow$ $\mathbb{R}$, that is,

$$
\|T x-T y\|^{2} \leq\|x-y\|^{2}+\lambda(y)\langle x-T x, y-T y\rangle, \quad \forall x, y \in C .
$$

(5.4.3) $F(T) \neq \varnothing$.

Then for any $x \in C$, the sequence $\left\{S_{n}(x)\right\}_{n \in \mathbb{N}}$ defined by

$$
S_{n}(x)=\frac{1}{n} \sum_{k=0}^{n-1} T^{k} x
$$

converges weakly to some point $v \in F(T)$.

\section{Author details}

${ }^{1}$ Center for General Education, Southern Taiwan University, 1 Nantai St., Yongkang Dist., Tainan 71005, Taiwan ${ }^{2}$ Nanjeon Institute of Technology, 178 Chaogin Rd., Yenshui Dist., Tainan 73746, Taiwan ${ }^{3}$ Fooyin University, 151 Jinxue Rd., Daliao Dist., Kaohsiung 83102, Taiwan ${ }^{4}$ Department of Industrial Management, National Pingtung University of Science and Technology, 1 Shuefu Rd., Neopu, Pingtung 91201, Taiwan

\section{Authors' contributions}

All authors read and approved the final manuscript.

\section{Competing interests}

The authors declare that they have no competing interests.

Received: 25 August 2011 Accepted: 23 December 2011 Published: 23 December 2011

\section{References}

1. Browder, FE: Fixed point theorems for noncompact mappings in a Hilbert space. Proc Nat Acad Sci USA. 53, 1272-1276 (1965). doi:10.1073/pnas.53.6.1272

2. Goebel, K, Kirk, WA: Topics in Metric Fixed Point Theory, Cambridge Studies in Advanced Mathematics. Cambridge University Press, Cambridge28 (1990)

3. lemoto, S, Takahashi, W: Approximating common fixed points of nonexpansive mappings and nonspreading mappings in a Hilbert space. Nonlinear Anal. 71, 2082-2089 (2009). doi:10.1016/j.na.2009.03.064

4. Kohsaka, F, Takahashi, W: Fixed point theorems for a class of nonlinear mappings related to maximal monotone operators in Banach spaces. Arch Math. 91, 166-177 (2008). doi:10.1007/s00013-008-2545-8

5. Takahashi, W, Yao, JC: Fixed point theorems and ergodic theorems for nonlinear mappings in Hilbert spaces. Taiwanese J Math. 15, 457-472 (2011)

6. Kocourek, P, Takahashi, W, Yao, JC: Fixed point theorems and weak convergence theorems for generalized hybrid mappings in Hilbert spaces. Taiwanese J Math. 14, 2497-2511 (2010)

7. Takahashi, W: Fixed point theorems for new nonlinear mappings in a Hilbert space. J Nonlinear Convex Anal. 11, 79-88 (2010)

8. Aoyama, K, lemoto, S, Kohsaka, F, Takahashi, W: Fixed point and ergodic theorems for $\lambda$-hybrid mappings in Hilbert spaces. J Nonlinear Convex Anal. 11, 335-343 (2010)

9. Butnariu, D, lusem, AN: Totally Convex Functions for Fixed Points Computation and Infinite Dimensional Optimization. Kluwer Academic Publishers, The Netherlands (2000)

10. Ciorãnescu, I: Geometry of Banach Spaces, Duality Mappings and Nonlinear Problems. Kluwer Academic Publishers, The Netherlands (1990)

11. Opial, Z: Weak convergence of the sequence of successive approximations for nonexpansive. mappings Bull Amer Math Soc. 73, 591-597 (1967). doi:10.1090/S0002-9904-1967-11761-0

doi:10.1186/1687-1812-2011-105

Cite this article as: Huang et al:: Fixed point and weak convergence theorems for point-dependent $\lambda$-hybrid mappings in Banach spaces. Fixed Point Theory and Applications 2011 2011:105. 